\title{
Phase Coherence Zones in Flight Simulation
}

\author{
P.M. Jonik, ${ }^{*}$ A.R. Valente Pais, ${ }^{\dagger}$ M.M. van Paassen, ${ }^{\ddagger}$ and M. Mulder ${ }^{\S}$ \\ Delft University of Technology, Delft, The Netherlands
}

\begin{abstract}
In flight simulation detailed knowledge of human motion perception is crucial. Phase differences between inertial and visual motion introduced by motion filters might have negative effects on the fidelity of flight simulation. This study investigated human visualvestibular phase-error detection. An experiment was conducted to measure the maximum amount of phase lead of the inertial motion with respect to the visual motion that remains undetected by the human. It contributes to the assessment of so-called coherence zones investigated in previous studies. Possible effects of the stimulus frequency, amplitude and the axis of rotation were examined. They were found to have no significant influence on phase-error detection. The average phase-error threshold was determined at 22 degrees. In accordance to previous studies the results showed that humans can be considered more like phase-error detectors rather than time delay detectors.
\end{abstract}

\section{Introduction}

While always matching in real life, in flight simulation visual and inertial motion are not necessarily always coherent. Moving base flight simulators have a limited motion space. The visual scene which is projected onto a screen and the accompanying inertial motion can be generated independently. In order to find a compromise between highest realism and the simulator limits, motion filters are used to calculate those inertial stimuli which best reflect the visual scene. ${ }^{1}$ All this is only possible since humans do not immediately detect mismatches between both stimuli. In fact there is a more or less broad range in which, though different, both motions seem to be coherent for the human pilot. ${ }^{2}$ This range was called "coherence zone" by Van der Steen. ${ }^{2}$

In his study Van der Steen examined mismatches between inertial and visual motion in terms of amplitude. While he kept the visual signal amplitude constant, he varied the amplitude of the inertial motion throughout the experiment. From this he could derive a lower and an upper threshold at which humans perceived the inertial motion as being too weak or too strong with respect to the visual stimulus. Those thresholds define a coherence zone which will be referred to as "amplitude coherence zone" throughout this paper.

Valente Pais et al. ${ }^{3}$ extended the work of Van der Steen to higher amplitudes of the visual stimulus. They found that for high visual amplitudes the coherence zone drops below the one-to-one line. This means that in flight simulation scenarios, humans prefer inertial motion amplitudes lower than the corresponding visual motion amplitude. This finding can be used for fine tuning flight simulator motion filters. For high amplitude stimuli the required motion space of the motion platform can be reduced, while still providing a realistic illusion of flight.

However, differences in amplitude are not the only possible kind of motion-visual mismatches. Grant and Lee $^{4}$ shifted identical sinusoidal stimuli in time, causing the inertial motion to lead the visual motion by a certain phase difference. By varying the amount of phase lead they could determine thresholds for the maximum phase difference that is not perceived by the human. The region containing all phase differences

\footnotetext{
*MSc. student, Control and Simulation Division, Faculty of Aerospace Engineering, P.O. Box 5058, 2600GB Delft, The Netherlands; peter.jonik@gmx.de.

$\dagger$ Ph.D. student, Control and Simulation Division, Faculty of Aerospace Engineering, P.O. Box 5058, 2600GB Delft, The Netherlands; a.r.valentepais@tudelft.nl. AIAA student member.

$\ddagger$ Associate Professor, Control and Simulation Division, Faculty of Aerospace Engineering, P.O. Box 5058, 2600GB Delft, The Netherlands; m.m.vanpaassen@tudelft.nl. AIAA member.

$\S$ Professor, Control and Simulation Division, Faculty of Aerospace Engineering, P.O. Box 5058, 2600GB Delft, The Netherlands; m.mulder@tudelft.nl. AIAA senior member.
} 
that remain undetected by the human may be said to define a "phase coherence zone". Grant and Lee examined those phase coherence zones for different experimental conditions, where the overall maximum phase difference was found to be 57 degrees.

Knowledge on phase coherence zones, as of amplitude coherence zones, allows for a better tuning of motion filter settings. Motion filters introduce a certain phase lead of the inertial motion with respect to the visual signal. ${ }^{1}$ It is of crucial importance to ensure that the resulting phase difference remains undetected by the pilot. ${ }^{5}$

The experiment described in this present paper aims at gathering further knowledge of phase coherence zones. The conditions tested were a combination of three frequencies and two amplitudes of the stimulus signal. The experimental conditions were chosen to extend Grant and Lee's work to higher amplitudes and frequencies. Also, a different axis of rotation was chosen: yaw. However, two conditions were performed with pitch motion in order to have a direct comparison with Grant and Lee's data.

Besides finding actual values for phase coherence zones for the conditions tested, the experiment mainly aimed at establishing a basis for a theoretical model which could be extrapolated for other amplitudes and frequencies. Hypotheses about how the experiment conditions were expected to impact on phase coherence zones were formulated and can be found in Section III.A.

The first section of this report presents the most important results from previous research that were considered relevant for this topic. It continues with describing the setup of the performed experiment in detail. Thereafter the results are presented and discussed. The report ends with a short conclusion summarizing the most important findings.

\section{Literature Review}

This section presents a short overview of literature concerning perception of self-motion and the measurement of coherence zones.

\section{II.A. Self-motion perception}

The human being has a variety of sensors which allow perception of self-motion. Van der Steen ${ }^{2}$ suggested a classification of the stimuli affecting human self-motion perception, where they can be splitted among two categories: inertial and environmental stimuli. Inertial stimuli refer to actual accelerations and velocities of a body with respect to an inertial reference frame, in other words, the actual motion a body is exposed to. The resulting forces and moments are mainly perceived by the vestibular system, however also proprioceptive and somatosensory systems contribute to this sensation. Environmental stimuli, on the other hand, describe the relative motion of the environment in which the body moves. Here, the visual system has a major contribution. However also auditory, proprioceptive and somatosensory systems can have small influence in perceiving environmental stimuli. A brief description of the vestibular and visual systems is done in the following sub-sections.

\section{II.A.1. Vestibular system}

The vestibular organ is responsible for perceiving linear and angular accelerations of the body. It consists of the semicircular canals and the otolith organ. Both are described in the next two sections. Figure 1 shows a schematic layout of the vestibular system.

Semicircular CANALS The semicircular canals allow the detection of angular accelerations in all three spatial axes. ${ }^{6}$ As can be seen in Figure 1 there are three semicircular canals with nearly orthogonal orientation to each other, hence, one canal per axis.

Each canal is filled with a fluid called endolymph. When turning the head, inertia causes the endolymph to move with respect to the canals. Each semicircular canal contains a gelatinous structure called cupula that is equipped with hair cells, the cilia. The moving endolymph causes the cupula to bend, which affects the discharge rate of the hair cells contained in it. ${ }^{7}$ This neuronal signals are sent to the brain, where they are interpreted and transformed to a perception of self-rotation.

When rotating with a constant speed for a longer period, the endolymph velocity will eventually become equal to the velocity of the canals, that is, the endolymph will not move with respect to the canal's wall and the cupula will not bend. This results in a decreasing and eventually disappearing perception of self-rotation. ${ }^{6}$ 


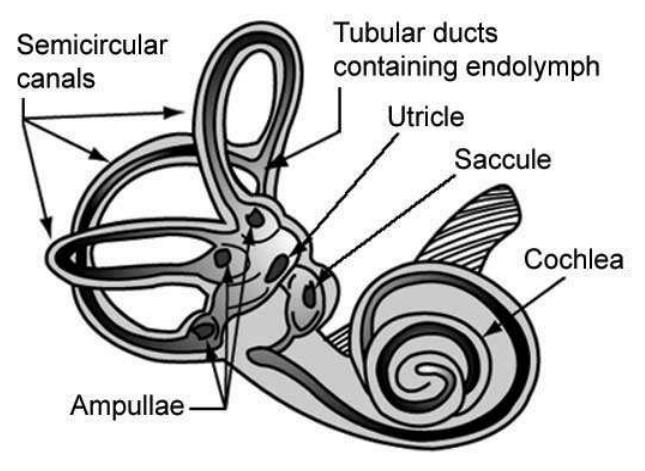

Figure 1. Schematic of vestibular system.

Van der Steen ${ }^{2}$ suggested models for the visual and vestibular systems where the transition from inertial or environmental stimuli to perception of self-motion is described. The structure of a model of angular acceleration is depicted in Figure 2. This model consists of the sensor dynamics (in this case the semicircular canals, $H_{S C C}$ ) and a neuronal filter, $N F_{S C C}$. The model of the semicircular canals $H_{S C C}$ describes the transition of the angular acceleration $\ddot{R}_{B I}$ of the body with respect to an earth-fixed reference frame to neuronal discharge rates. This neuronal output is then sent to the neuronal filter, $N F_{S C C}$, where it is translated to a perceptual variable, $\hat{R}_{B I}$. $\hat{R}_{B I}$ therefore describes a subjective sensation of rotation resulting from the angular acceleration, $\ddot{R}_{B I}$, when the semicircular canals are the only organ involved.

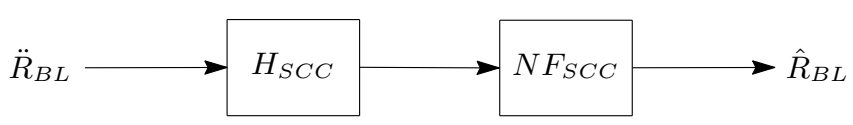

Figure 2. Signal processing from angular accelerations with respect to the body to perceived self-motion.

The dynamics of the semicircular canals have been examined in various studies. Fernandez and Goldberg, ${ }^{8}$ for example, made measurements of neuronal activity of squirrel monkeys and could extend an existing model to a second-order filter model with a lead term. Hosman and Van der Vaart ${ }^{7}$ conducted psychophysical experiments to determine perception thresholds and showed that their results could be described by a very similar model. Apparently, the dynamics of the semicircular canals alone and the overall perceptual model were nearly identical for the conditions examined. This fact, on one hand confirms the findings of Fernandez and Goldberg, and on the other hand gives an indication of what the neuronal filter, $N F_{S C C}$, might be.

The transfer function, $H_{S C C}$, found by Hosman and Van der Vaart is given in Equation (1). It relates the input angular acceleration, $\ddot{R}_{B I}$, to the output neuronal discharge rate, $n_{d i s, S C C}$.

$$
H_{S C C}(j \omega)=\frac{n_{d i s, S C C}}{\ddot{R}_{B I}}=\frac{(1+0.11 j \omega)}{(1+5.9 j \omega)(1+0.005 j \omega)}
$$

The overall transfer function of the semicircular canals, $Y_{S C C}$, is accordingly $Y_{S C C}=H_{S C C} N F_{S C C}$. It directly relates the angular acceleration, $\ddot{R}_{B I}$, to the perceptual variable, $\hat{R}_{B I}$. Considering that Fernandez and Goldberg and Hosman and Van der Vaart found a similar model for the semicircular canals' dynamics alone and for the overall perception model including the neuronal filter, implies that the neuronal filter, $N F_{S C C}$, is a pure gain when measuring angular acceleration perception thresholds. ${ }^{2}$

Mathematically, the input to the semicircular canals can also be represented in terms of angular velocity $\dot{R}_{B I}$, where $\dot{R}_{B I}=\frac{\ddot{R}_{B I}}{j \omega}$. The resulting transfer function, $H_{S C C, \dot{R}}$, relating angular velocity to neuronal discharge rates is shown in Equation (2).

$$
H_{S C C, \dot{R}}(j \omega)=\frac{n_{d i s, S C C}}{\dot{R}_{B I}}=\frac{n_{d i s, S C C}}{\frac{\ddot{R}_{B I}}{j \omega}}=j \omega \frac{(1+0.11 j \omega)}{(1+5.9 j \omega)(1+0.005 j \omega)}=j \omega H_{S C C}(j \omega)
$$


Figure 3 shows a bode plot of the semicircular canals' dynamics represented in terms of velocity input, $H_{S C C, \dot{R}}$. It is noteworthy that the magnitude is approximately constant for a frequency range which lies in the natural range of human motion. In other words, the human can be considered a nearly perfect velocity sensor between about 0.5 and $5 \mathrm{rad} / \mathrm{s}$.

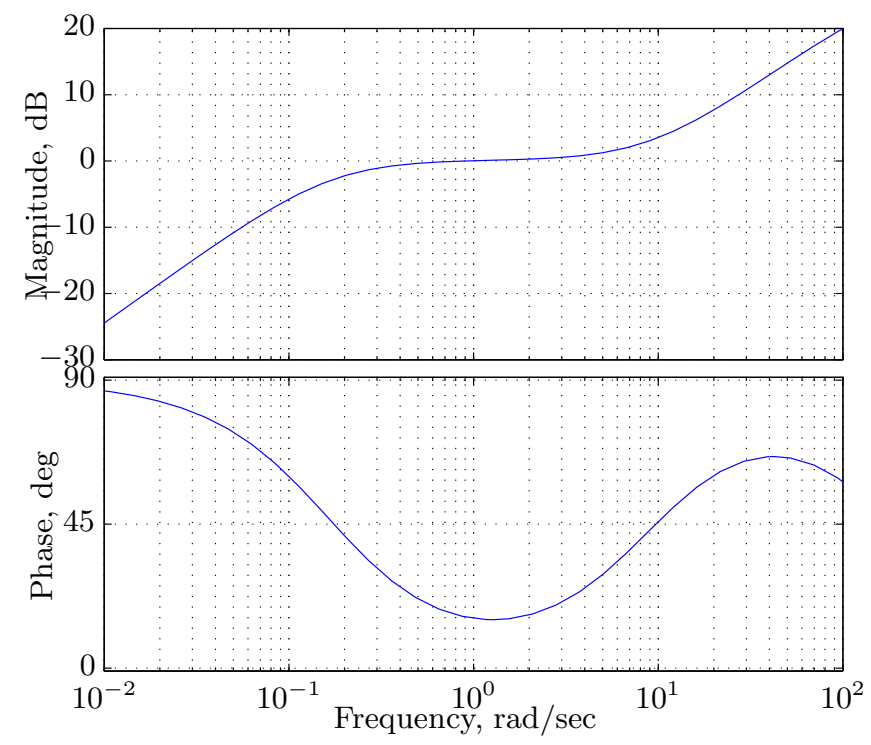

Figure 3. Semicircular canal model dynamics in terms of perceived velocity output, $H_{S C C, \dot{R}}$.

OTOLITH ORGAN The otolith organ is responsible for detecting linear acceleration in all three spatial axes. Otholiths are small solid calcium carbonate particles located in the saccule and utricle (see Figure 1). Together they make the otolith organ. The otoliths are connected to small hair cells. When the body is accelerated, inertia causes the otholits to bend those hair cells. The stimulated hair cells send neuronal signals to the brain where they are interpreted and transformed into a perception of linear self-motion. ${ }^{6,7}$

The model of neuronal filters as described in Section II.A.1 also applies here. However here, the model relates the linear acceleration of the body, $\ddot{X}_{B I}$, to a perception of linear self-motion, $\hat{X}_{B I}$.

Hosman and Van der Vaart ${ }^{7}$ suggested a simple second-order lead-lag model for the dynamics of the otholith organ, $H_{O T O}$, which is expressed by Equation (3). It relates the neuronal discharge rates caused by the otolith organ, $n_{\text {dis }, \text { OTO }}$, to the linear acceleration input, $\ddot{X}_{B I}$.

$$
H_{\text {OTO }}(j \omega)=\frac{n_{\text {dis }, \text { OTO }}}{\ddot{X}_{B I}}=\frac{1+j \omega}{(1+0.5 j \omega)(1+0.016 j \omega)}
$$

\section{II.A.2. Visual system}

The visual system detects motion in the environment and even in absence of inertial motion the visual system can induce a realistic sensation of self-motion. A well known example for this is the so-called "train-illusion", described by Van der Steen:

"When one is sitting in a train and an adjacent train starts to move, one may perceive self-motion, while the adjacent train is perceived stationary."

This phenomenon is called vection. ${ }^{9}$ Vection is called saturated as soon as the environment is perceived to be stationary while only the body itself seems to move. ${ }^{10}$ The state of saturated vection can be reached when the visual scene shows either translatory motion at a constant velocity, or yaw motion at a constant angular velocity. ${ }^{2}$ Both motions have in common that there is an absence of linear and angular accelerations 
of the body. Furthermore, the degree of realism of the presented visual scene plays an important role. The probability of vection to saturate increases with a more realistic scenery. ${ }^{11}$

The neuronal filter model, as presented in Section II.A.1, can also be used here (see Figure 4). However, the input consists of position and orientation of the visual scene with respect to the body, which are expressed by $X_{E B}$ and $R_{E B}$, respectively.

The dynamics of the visual system, $H_{V I S}$, are usually modeled as a pure time delay of tens of milliseconds. $^{2}$ The neuronal filter, $N F_{V I S}$, is responsible for assigning the polarity and structure of the visual scene. ${ }^{2}$

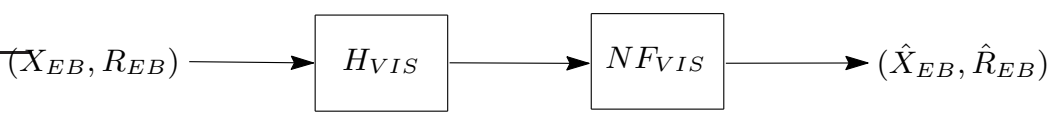

Figure 4. Signal processing from visual environment with respect to the body to perceived self-motion.

\section{II.B. Amplitude coherence zones}

The following sections present results of amplitude coherence zone experiments by Van der Steen ${ }^{2}$ and Valente Pais et al. ${ }^{3}$

\section{II.B.1. Van der Steen}

Van der Steen ${ }^{2}$ investigated amplitude coherence zones for roll and yaw motion. The stimulus profile used was a smoothed step-like acceleration signal. While the visual amplitude was held at a fixed value, the inertial amplitude was varied between runs using a staircase algorithm. Subjects were asked if they perceived the outside world to be stationary or not. A non-stationary outside world would indicate a perceived mismatch between the inertial and visual cues amplitude.

He found that a region exists above and below the one-to-one match between both stimuli that is still perceived to be coherent. He called this region "coherence zone". The coherence zone is bounded by an upper and a lower threshold that mark the largest and smallest inertial amplitudes, respectively, that are still perceived as coherent with the visual stimulus amplitude.

The coherence zone can be expressed mathematically by defining a coherence zone width (CZW) and a point of mean coherence (PMC). ${ }^{2}$ The coherence zone width describes the broadness of the region where inertial and visual motion seem to match for the human observer and can be calculated from the corresponding upper and lower thresholds (see Equation (4)). The point of mean coherence represents the mean value of the coherence zone and is calculated using Equation (5).

$$
\begin{gathered}
C Z W=t h_{\text {upper }}-t h_{\text {lower }} \\
P M C=t h_{\text {lower }}+\frac{C Z W}{2}
\end{gathered}
$$

\section{II.B.2. Valente Pais et al.}

Valente Pais et $a l .^{3}$ extended the work of Van der Steen for higher amplitudes. Also the question asked was changed. Subjects were not asked if they perceived the outside world to be stationary, but had to judge directly if both stimuli felt coherent or not. It was found that the coherence zone width became larger with rising amplitude of the visual profile. In addition, for very high amplitudes the coherence zone completely dropped below the one-to-one line. The upper and lower thresholds found by Valente Pais et al. are shown in Figure 5, together with data form Van der Steen.

Furthermore Valente Pais et al. also investigated an influence of the stimulus frequency on yaw coherence zones. A similar experimental setup was used, however the stimuli were replaced by sinusoidal acceleration profiles with amplitudes of $12 \mathrm{deg} / \mathrm{s}^{2}$ and $30 \mathrm{deg} / \mathrm{s}^{2}$ and frequencies of $2 \mathrm{rad} / \mathrm{s}$ and $10 \mathrm{rad} / \mathrm{s}$. The results showed a significant decrease in the point of mean coherence for rising frequency. This was explained using 


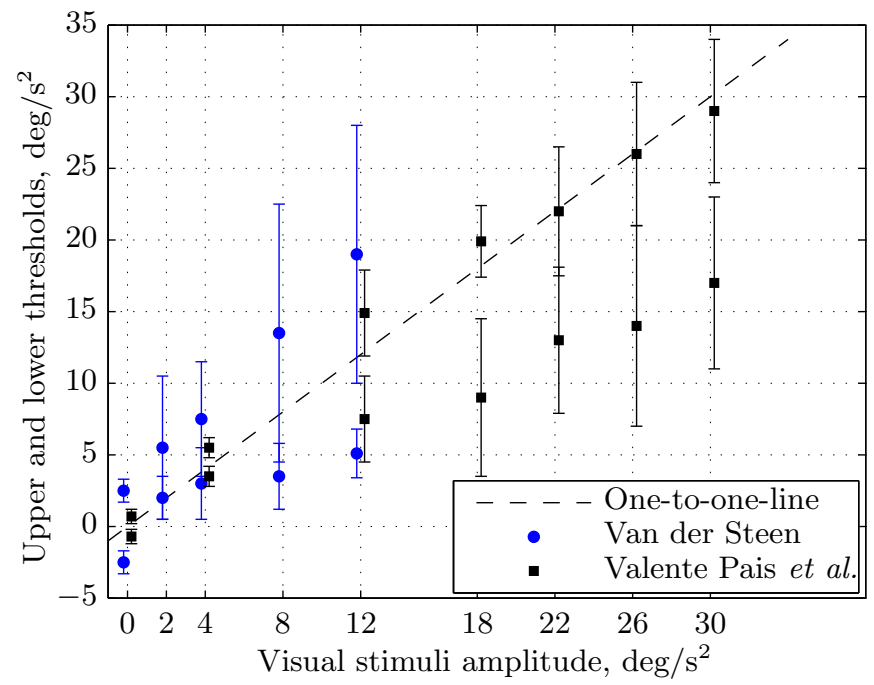

Figure 5. Amplitude coherence zones obtained by Van der Steen ${ }^{2}$ and Valente Pais et al. ${ }^{3}$

the dynamics of the semicircular canals. Figure 6 shows the magnitude of the semicircular canals model presented in Equation (2). It relates angular velocity input to perceived velocity output, while the neuronal filter is assumed to be a pure gain. The lines mark the frequencies used during the experiment.

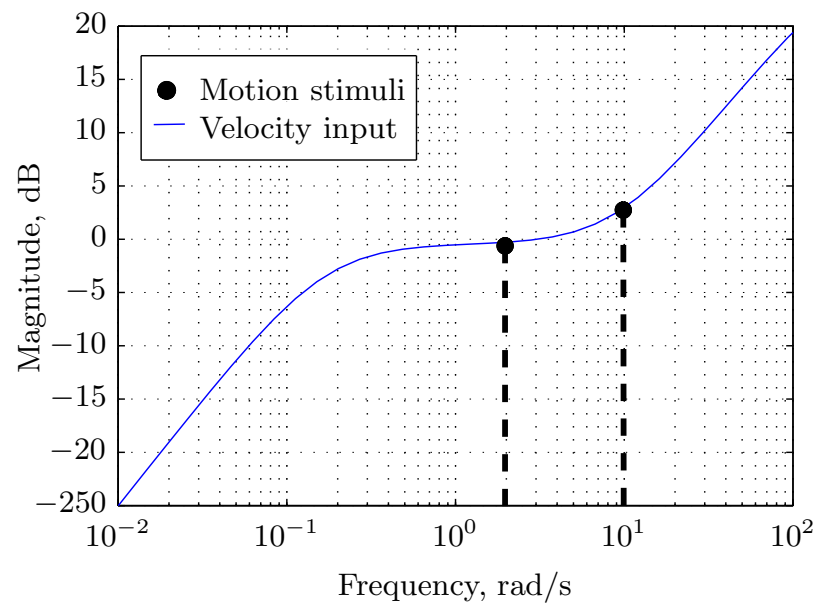

Figure 6. Effect of frequency on amplitude coherence zones caused by the semicircular canals dynamics

As can be seen, the magnitude of the semicircular canals is significantly higher for $10 \mathrm{rad} / \mathrm{s}$ than for $2 \mathrm{rad} / \mathrm{s}$. This means that at the high frequency condition the angular velocity perceived by the vestibular system is higher than at the lower frequency, although the amplitude is the same for both conditions. This might have induced the participants to prefer lower inertial amplitudes at the higher frequency when judging the visual-vestibular coherence, leading to a decrease of the point of mean coherence. This implies that there is no accurate internal representation of the semicircular canals dynamics that would allow its effect to be compensated for during the comparison of visual and inertial cues. 


\section{II.C. Phase coherence zones}

\section{II.C.1. Grant and Lee}

Grant and $\mathrm{Lee}^{4}$ performed an experiment measuring visual-vestibular phase-error detection. Although not stated by the authors this can be interpreted as a measurement of phase coherence zones. However, unlike for amplitude coherence zones, it does not make much sense to measure upper and lower phase thresholds. As mentioned before, the motion filters driving the simulator motion platform introduce a phase lead of the inertial signal with respect to the visual signal. Thus, a lag of the inertial stimulus practically does not occur. The lower threshold is therefore set at zero degrees of phase difference. Accordingly, the phase coherence zone width can be directly specified by the absolute value of the upper phase-error threshold. This upper threshold is the minimum phase-error which humans are able to detect consistently.

Grant and Lee determined the upper phase-error threshold during pitch motion. They investigated effects of frequency and amplitude of the stimulus profile, as well as effects of motion gain and scenery complexity. The conditions are summarized in Table 1.

Table 1. Experimental factors and levels used by Grant and Lee.

\begin{tabular}{lcc}
\hline \hline Factor & High & Low \\
\hline Frequency & $6.283 \mathrm{rad} / \mathrm{s}$ & $1.257 \mathrm{rad} / \mathrm{s}$ \\
Amplitude (pitch velocity) & $5.73 \mathrm{deg} / \mathrm{s}$ & $2.29 \mathrm{deg} / \mathrm{s}$ \\
Motion gain & 1.0 & 0.5 \\
Scene complexity & High & Low \\
\hline \hline
\end{tabular}

The visual display was generated using a helmet-mounted display. The resulting synchronization errors that were caused by the head tracker were taken into account. The phase-error threshold was determined using a modified Levitt method. ${ }^{12}$ This psychophysical method converges to a value, where the probability of detecting the phase error is $79 \%$. Subjects were shown two intervals, one coherent and one with a phase error, and were asked to identify the coherent interval. The amount of phase error introduced in each interval varied during the experiment and depended on the subjects' answers to the previous interval using a 1-up-3-down algorithm. ${ }^{4}$

Table 2. Phase error thresholds obtained by Grant and Lee.

\begin{tabular}{|c|c|c|c|c|c|c|c|c|c|}
\hline & Visual complexity & Low & Low & High & High & Low & Low & High & High \\
\hline Amplitude & Motion gain & 0.5 & 0.5 & 0.5 & 0.5 & 1 & 1 & 1 & 1 \\
\hline \multirow[t]{2}{*}{$2.29 \mathrm{deg} / \mathrm{s}$} & Frequency $(\mathrm{rad} / \mathrm{s})$ & 1.257 & 6.283 & 1.257 & 6.283 & 1.257 & 6.283 & 1.257 & 6.283 \\
\hline & Phase threshold (deg) & 88 & 68 & 82 & 60 & 71 & 53 & 56 & 72 \\
\hline \multirow{4}{*}{$\begin{array}{l}\text { Amplitude } \\
5.73 \mathrm{deg} / \mathrm{s}\end{array}$} & Visual complexity & Low & Low & High & High & Low & Low & High & High \\
\hline & Motion gain & 0.5 & 0.5 & 0.5 & 0.5 & 1 & 1 & 1 & 1 \\
\hline & Frequency $(\mathrm{rad} / \mathrm{s})$ & 1.257 & 6.283 & 1.257 & 6.283 & 1.257 & 6.283 & 1.257 & 6.283 \\
\hline & Phase threshold (deg) & 51 & 49 & 46 & 44 & 40 & 43 & 43 & 48 \\
\hline
\end{tabular}

Table 2 lists the measured phase-error thresholds for all conditions. The values were taken from Grant and Lee's report and rounded to integer values. A high significance of motion amplitude can be seen, as higher amplitudes lead to lower detection thresholds. The motion gain showed a significant influence for a frequency of $1.257 \mathrm{rad} / \mathrm{s}$ or at low visual complexity. Frequency was only significant for a motion gain of 0.5 or with low visual complexity. It is remarkable that all but one condition in Table 2 with an amplitude of $2.29 \mathrm{deg} / \mathrm{s}$ show a mean reduction of approximately $20 \mathrm{deg}$ from $1.257 \mathrm{rad} / \mathrm{s}$ to $6.283 \mathrm{rad} / \mathrm{s}$. The mean upper phase-error threshold, or phase coherence zone width was found to be $57 \mathrm{deg}$.

\section{Experiment}

The experiment in this study aimed at extending the experiment of Grant and Lee ${ }^{4}$ to higher amplitudes and frequencies. The following sections contain hypotheses about the experiment outcome and a detailed descriptions of the methods used to conduct the experiment. 


\section{III.A. Hypotheses}

Two different outcomes could be expected from the experiment. On the one hand, humans may act more like phase error detectors and not like time delay detectors. That means that the actual time delay between visual and inertial motion which is recognized by the human would vary with the stimulus frequency, while the phase error threshold would remain fairly constant. On the other hand, if subjects would act more like time delay detectors, the phase-error threshold would vary with stimulus frequency while the time delay would remain constant. Figure 7 shows the idealized outcomes for either case.
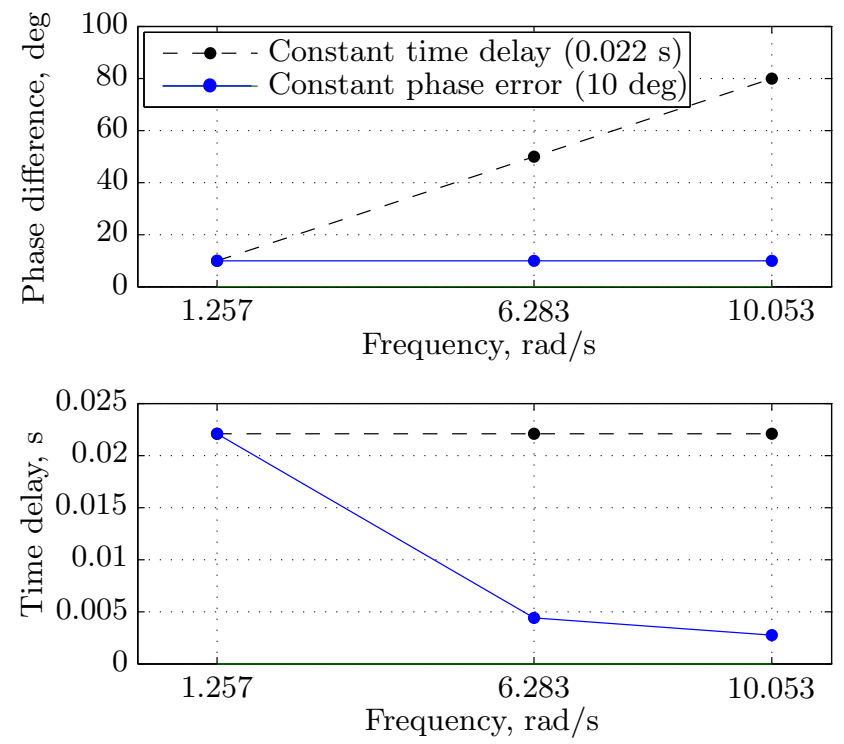

Figure 7. Idealized curves for perfect phase error sensors and perfect time delay sensors.

A third option is possible, where subjects act like phase detectors but the phase-error threshold depends on frequency but not in a way that results in a constant time delay. Based on the work of Grant and Lee ${ }^{4}$ two contradicting hypotheses regarding the frequency dependency were formulated. Both were based on their data, where two opposing effects of frequency on phase-error thresholds could be found. The results of the present experiment were expected to confirm one of those hypotheses:

1. This first hypothesis is related to the theory of the imperfect internal representation of the semicircular canals used to explain the decrease of the point of mean coherence at the higher frequency for the amplitude coherence zones (c.f. Section II.B). As shown in Table 2 the low amplitude conditions show a decrease in phase-error threshold from the low to the high frequency of approximately 20 degrees. There is an exception for the condition with high visual complexity and motion gain of one. Looking at Figure 8 one can see that the phase of the semicircular canals dynamics increases by about $20 \mathrm{deg}$ between those frequencies. Hence, the hypothesis posed is that for a fixed amplitude there is one constant internal phase-error threshold for all frequencies below which phase differences remain undetected by the human. This implies that the neuronal filter of the semicircular canals is a pure gain (Section II.A.1) and the dynamics of the semicircular canals contribute to this internal phase error. Therefore, the measured phase-error thresholds are the sum of the introduced phase error and the phase lead of the semicircular canals' dynamics $H_{S C C}$. In a frequency range between $1 \mathrm{rad} / \mathrm{s}$ to $13 \mathrm{rad} / \mathrm{s}$ this would result in a reduction of the phase-error thresholds with rising frequency.

2. The second hypothesis is based on the conditions with an amplitude of $5.73 \mathrm{deg} / \mathrm{s}$. Table 2 shows that for those conditions there was nearly no influence of frequency on phase-error thresholds. To explain this, the hypothesis posed is that the neuronal filter contains the inverse of the semicircular canals' phase dynamics. To put it another way, the brain "knows" the behavior of the semicircular canals and compensates for its dynamics. Consequently the dynamics of the semicircular canals have no significant influence on the perception of phase-error thresholds. As a result, all phase-error thresholds are supposed to remain more or less constant for all frequencies. 


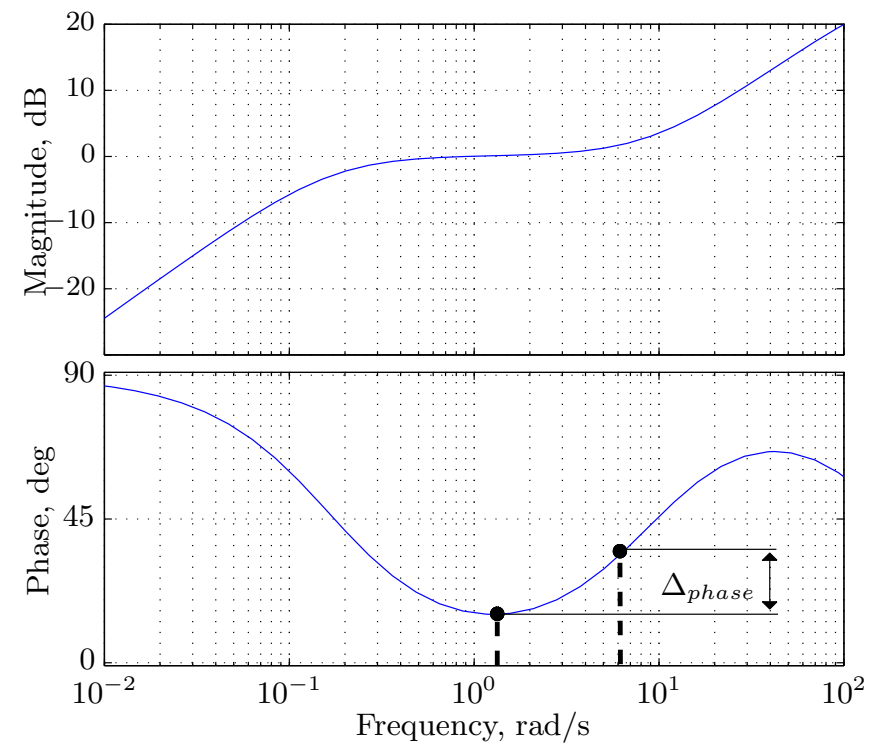

Figure 8. Semicircular canal model dynamics with both frequencies used by Grant and Lee. ${ }^{4}$ The phase difference introduced by the semicircular canals' dynamics is approximately 20 degrees.

Regarding the amplitude dependency of the phase thresholds it was hypothesized, that higher amplitudes would lead to smaller thresholds. Such an effect was also found in the data of Grant and Lee. It might be that at higher amplitudes the detection of errors is easier and more reliable. Therefore, for a constant frequency the phase-error thresholds were expected to become smaller with rising amplitude. This effect, however, was assumed to saturate above a certain amplitude higher than $5.73 \mathrm{deg}$.

Phase-error thresholds during pitch motion were expected to show the same trends as in yaw motion. However, they were expected to be lower because during pitch motion not only the semicircular canals but also the otolith organ is stimulated to a certain extent. For the examined pitch conditions, the resulting surge acceleration was $0.78 \mathrm{~m} / \mathrm{s}^{2}$ for the low frequency and $0.16 \mathrm{~m} / \mathrm{s}^{2}$ for the higher frequency. Both values are above the vestibular perception threshold for surge motion at the respective frequencies. Those thresholds are given in Heerspink et al., ${ }^{13}$ and range from about $0.03 \mathrm{~m} / \mathrm{s}^{2}$ to $0.05 \mathrm{~m} / \mathrm{s}^{2}$.

\section{III.B. Method}

The method used in this experiment was a staircase method based on the one used in the study of Valente Pais et al. ${ }^{3}$. The following sections describe the setup in detail and focus on the differences with respect to the method of Valente Pais et al. .

\section{III.B.1. Apparatus}

The experiment was conducted in the Simona Research Simulator (SRS) of the Delft University of Technology. The SRS provides motion in six degrees of freedom using a hydraulically driven hexapod type motion base. It allows a maximum displacement of \pm 41.6 degrees in yaw and $+23.7 /-24.3$ degrees in pitch. The SRS is equipped with an Inertial Measurement Unit consisting of three rate sensors and three accelerometers to measure all specific forces and rotational accelerations. ${ }^{14}$ The outside visual is projected onto a semicircular screen behind a collimating mirror, providing a field of view of 180 deg horizontal by 40 deg vertical. Three projectors with a resolution of $1280 \times 1024$ pixels and a refresh rate of $60 \mathrm{~Hz}$ are used to project the image. ${ }^{15}$ The visual scene used for the experiment showed a view of Schiphol airport form a viewpoint height of 5 meters. The scene included a grass field, part of a runway, a control tower and some lower buildings. 


\section{III.B.2. Experimental Conditions}

The experiment consisted of eight conditions, six in yaw and two in pitch. The two pitch conditions were only used for a direct comparison to Grant and Lee's data, whereas the six yaw conditions formed a full factorial repeated measures design.

All conditions are listed in Table 3.

Table 3. Experiment conditions.

\begin{tabular}{lcccccccc}
\hline \hline Rotation & Yaw & Yaw & Yaw & Yaw & Yaw & Yaw & Pitch & Pitch \\
Amplitude (deg/s) & 5.73 & 5.73 & 5.73 & 9.17 & 9.17 & 9.17 & 5.73 & 5.73 \\
Frequency $(\mathrm{rad} / \mathrm{s})$ & 1.257 & 6.283 & 10.053 & 1.257 & 6.283 & 10.053 & 1.257 & 6.283 \\
\hline \hline
\end{tabular}

Both lower frequencies of $1.257 \mathrm{rad} / \mathrm{s}$ and $6.283 \mathrm{rad} / \mathrm{s}$ were the same as used by Grant and Lee. The highest frequency was chosen to be in the range above $10 \mathrm{rad} / \mathrm{s}$ where the semicircular canals dynamics introduce a larger phase lead, but not too high frequency that it would become uncomfortable for the subjects in the simulator. The low amplitude chosen of $5.73 \mathrm{deg} / \mathrm{s}$ was also used by Grant and Lee. A higher amplitude of $9.17 \mathrm{deg} / \mathrm{s}$ was chosen to extend Grant and Lee's data to higher amplitudes.

The specific values of $9.17 \mathrm{deg} / \mathrm{s}$ for the amplitude and $10.053 \mathrm{rad} / \mathrm{s}$ for the frequency were selected such that some conditions would also produce identical amplitudes in acceleration. Initially, this was thought to provide an interesting comparison. Although the idea was discarded, the values remained since they lie within the range of interest. Table 4 shows the resulting amplitudes in acceleration, velocity and attitude for all conditions. The amplitude defining a condition is denoted in velocity. All conditions were chosen to produce reasonable values of acceleration and stay within the limits of SRS.

Every subject repeated every condition three times, which resulted in a total of 24 conditions per subject.

Table 4. Attitude and acceleration amplitudes for corresponding experiment conditions.

\begin{tabular}{ccccc}
\hline \hline & & \multicolumn{2}{c}{ Frequency, rad/s } \\
& 1.257 & 6.283 & 10.051 \\
\cline { 2 - 5 } Velocity amplitude & Attitude: $4.56 \mathrm{deg}$ & Attitude: $0.91 \mathrm{deg}$ & Attitude: $0.57 \mathrm{deg}$ \\
$5.73 \mathrm{deg} / \mathrm{s}$ & Acceleration: $7.20 \mathrm{deg} / \mathrm{s}^{2}$ & Acceleration: $36.00 \mathrm{deg} / \mathrm{s}^{2}$ & Acceleration: $57.60 \mathrm{deg} / \mathrm{s}^{2}$ \\
\hline Velocity amplitude & Attitude: $7.30 \mathrm{deg}$ & Attitude: $1.46 \mathrm{deg}$ & Attitude: $0.91 \mathrm{deg}$ \\
$9.17 \mathrm{deg} / \mathrm{s}$ & Acceleration: $11.52 \mathrm{deg} / \mathrm{s}^{2}$ & Acceleration: $57.60 \mathrm{deg} / \mathrm{s}^{2}$ & Acceleration: $92.17 \mathrm{deg} / \mathrm{s}^{2}$ \\
\hline \hline
\end{tabular}

\section{III.B.3. Subjects}

Eight subjects participated in the experiment, seven male and one female. Their ages were between 23 and 26 years with a mean of 24.75 years. Five subjects indicated previous experience with full motion simulators.

All subjects were briefed immediately before the start of the experiment. The briefing contained an explanation of the experiment setup, their task and some security instructions. The subjects were told that the experiment was divided in a number of blocks. Each block consisted of a random number of runs, of which some contained a phase error and others not. The number of runs with or without phase error was also stated to be randomized, however not in a normally distributed way. Although not explicitly told, it was suggested that their answers had no influence on the following run. All subjects were instructed to concentrate solely on phase-error detection. They were asked to avoid getting distracted by other clues and not to pay attention to, for example, possibly perceived amplitude mismatches. However, they were asked to report any kind of distractions to the experiment supervisor. They were also asked to report all of their impressions and describe their strategy.

Before the actual experiment started, three chosen conditions were used as test runs in order to give the subject time to get used to the procedure and to clarify potential questions.

\section{III.B.4. Procedure}

The subjects were placed in the right-hand-side pilot seat, their head being in the center of rotation. They wore a headset with active noise cancellation to assure that no sound from the motion system could provide 
any extra clue. For every subject the order of conditions was randomized before the experiment. All runs within one condition are referred to as a block in the following. One block contained several runs. The algorithm used to determine the phase error of each run was based on the subjects' answers. It was an an up-down staircase algorithm similar to the one used in Valente Pais et al. ${ }^{3}$. The initial phase difference was randomized between 0 and $5 \mathrm{deg}$. The initial step size was $8 \mathrm{deg}$. After each run subjects were asked if the inertial and visual cues were synchronized in time. They provided the answer using two buttons on a sidestick. Depending on the subject's answer the phase difference in the next run was either increased by the step size (if the answer was "yes") or decreased by the step size (if the answer was "no"). After each reversal in the subject's answer the step size was halved. If the same answer was given more than four times in a row the step size was doubled to assure a faster approach to the threshold. While there was no upper limit, the lower limit for the phase error was $0 \mathrm{deg}$. If the value reached zero and the subject answered "no" for two times in a row, the phase error in the next run was randomized between 50 - 70 deg. This assured that subjects who perceived phase errors although there were none could "restart" their judgment. Figure 9, Figure 10 and Figure 11 show a schematic of the staircase-method for three different runs with corresponding sequence of answers. A block was finished when either the step size reached 1 deg or the number of reversals reached ten.

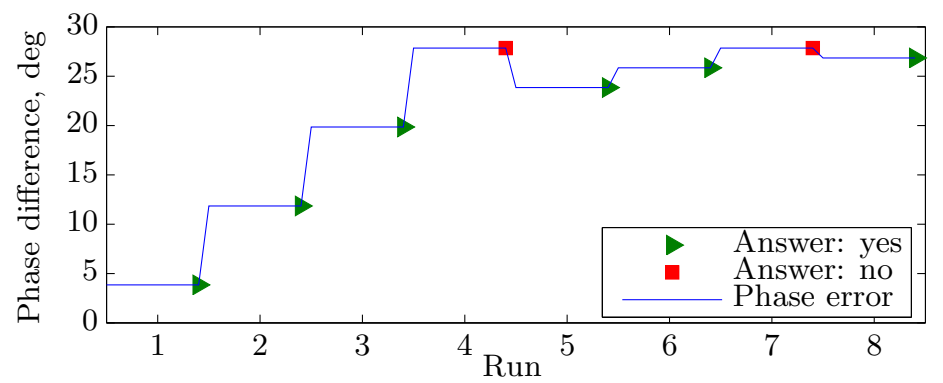

Figure 9. Example of a block of runs where the subjects converged relatively fast to the threshold value.

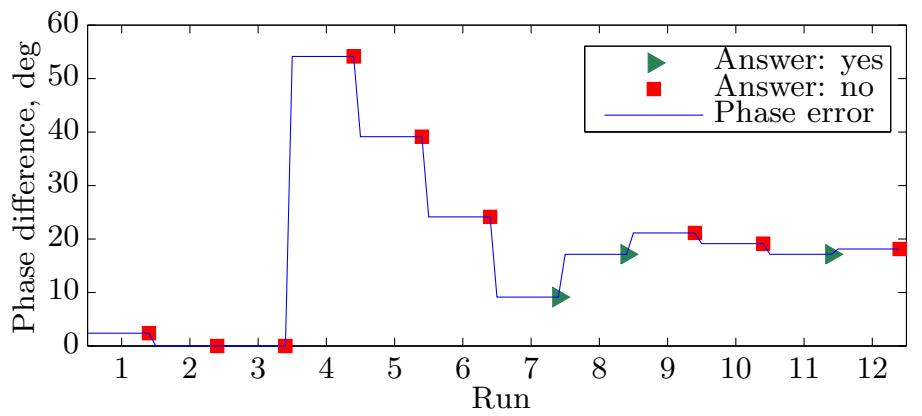

Figure 10. Example of a block of runs with a "restart" at high phase-error values between runs 3 and 4 .

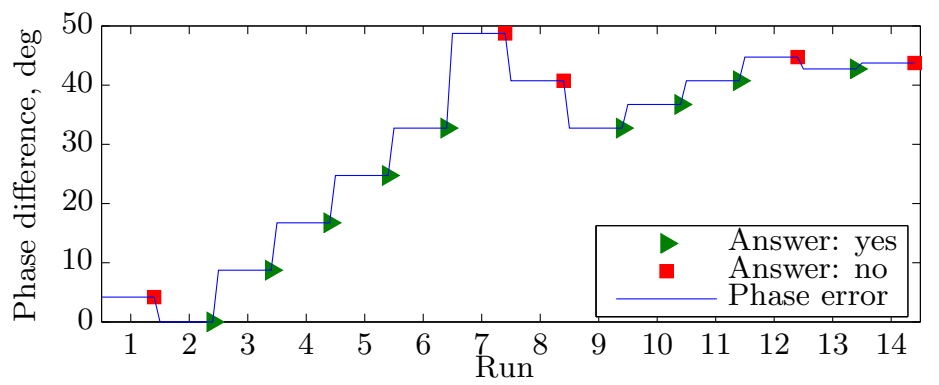

Figure 11. Example of a block of runs where the step size was doubled between runs 6 and 7 . 
Throughout the whole experiment subjects could talk to the supervisor using a headset. It was possible to make a break at any point of the experiment. Most subjects requested a break of about twenty minutes after two thirds of the conditions. The experiment duration was approximately three hours per subject.

\section{III.B.5. Motion Profile}

A sinusoidal motion profile was used for all experimental conditions. The maximum amplitude of the motion velocity was used to define the conditions shown in Table 3. Figure 12 shows the motion profile for a frequency of $1.257 \mathrm{rad} / \mathrm{s}$.

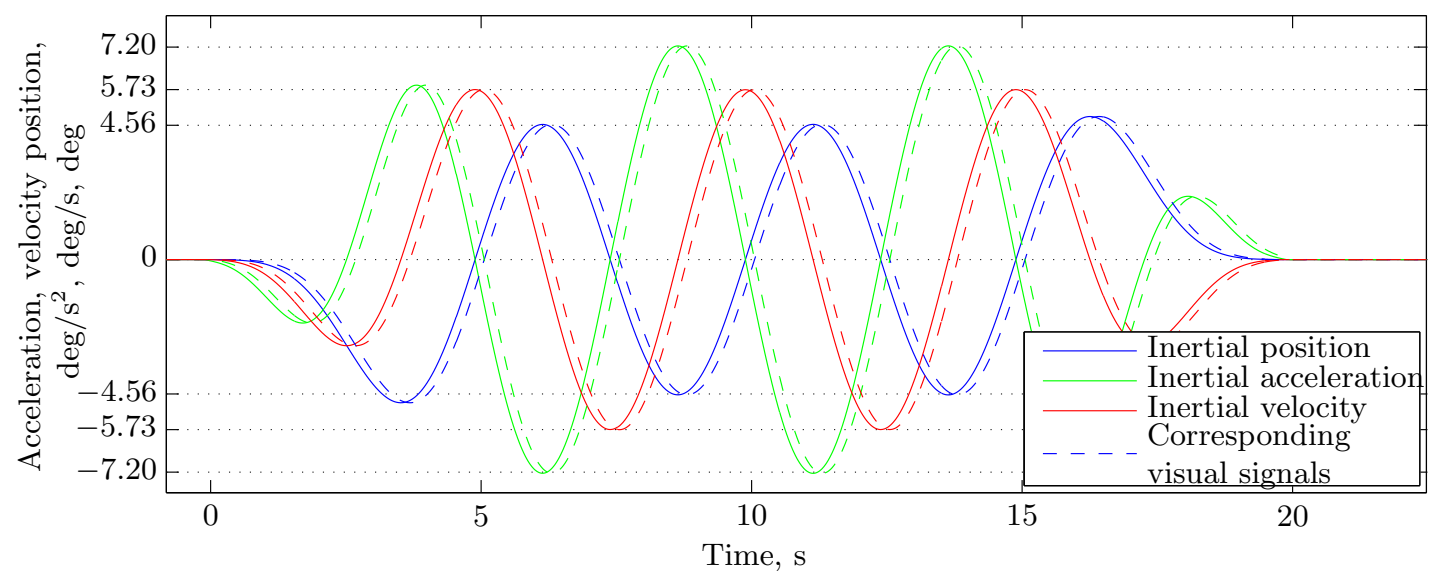

Figure 12. Acceleration, velocity and position signals for a frequency of $1.257 \mathrm{rad} / \mathrm{s}$ and corresponding visual signals with a phase lag.

A different number of periods was used, depending on the stimulus frequency. To guarantee a run time of at least $10 \mathrm{~s}$ for all frequencies, the number of periods had to be different for every frequency. The lowest frequency contained four periods, the mid frequency ten, and the highest frequency sixteen periods. In each case one period was used to fade the motion in and one period to fade it out. The fading was done as described in Valente Pais et al. .

In each run, the inertial motion consistently lead the visual motion by a constant amount of phase difference. By keeping a constant phase difference also during the fade-in, the inertial motion started to grow while the visual scene was still stationary, and during fade-out the visual motion kept on moving, while the inertial motion had already stopped. Although the inertial motion was considered to be sub-threshold during this short period, it could not be excluded that this fact might cause subjects to detect a time delay between the start of the visual motion and the start of the inertial motion. To avoid this additional clue, the visual scene was also faded-in. A blank screen was shown at the beginning of inertial motion, and only after a half period the visual scene would appear. To assure a soft transition between the blank screen and the visual scene, the visual was faded in during $500 \mathrm{~ms}$.

To guarantee an accurate relation between visual and inertial motion, known calibration data of the SRS was used. ${ }^{16}$ Taking into account the intrinsic time delay of the visual and motion systems of the SRS, the visual scene had to be artificially delayed by additional $6 \mathrm{~ms}$ to match the inertial motion.

\section{Results}

In order to convert the answers of each run to a distinct threshold value, psychometric curves were fitted. This was accomplished by assigning a value of zero to the answer "yes" and a value of one to the answer "no". The psychometric curves were then fitted using a least squares method, based on a normally distributed cumulative probability function. ${ }^{3}$ Figure 13 shows an example of such a fitted curve. The shape of a psychometric curve can be clearly characterized by two values: the mean and the standard deviation. Since the up-down staircase method used for this experiment aimed on finding a good estimate of the $50 \%$ probability of detection level, the mean of the psychometric curve was taken as the threshold value. 


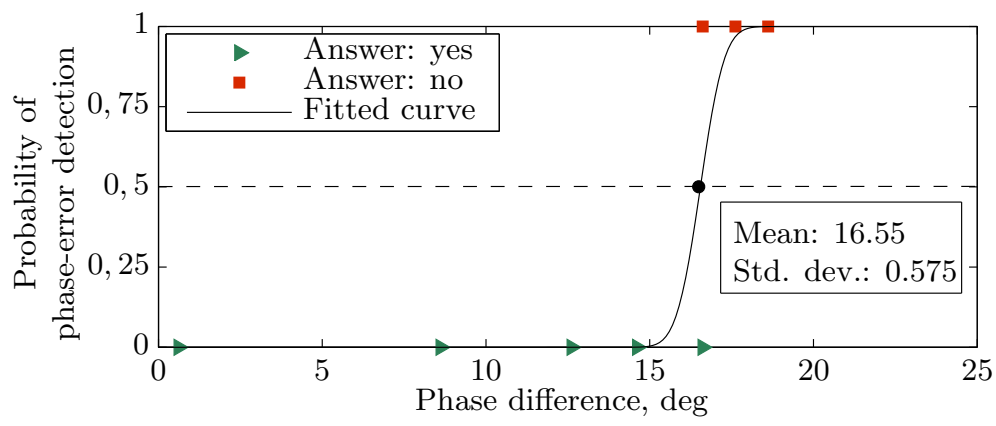

Figure 13. Fitted psychometric curve

The threshold values determined through the fitting were then averaged for all subjects and repetitions. Figure 14 shows the mean thresholds for all conditions represented in terms of phase and time.

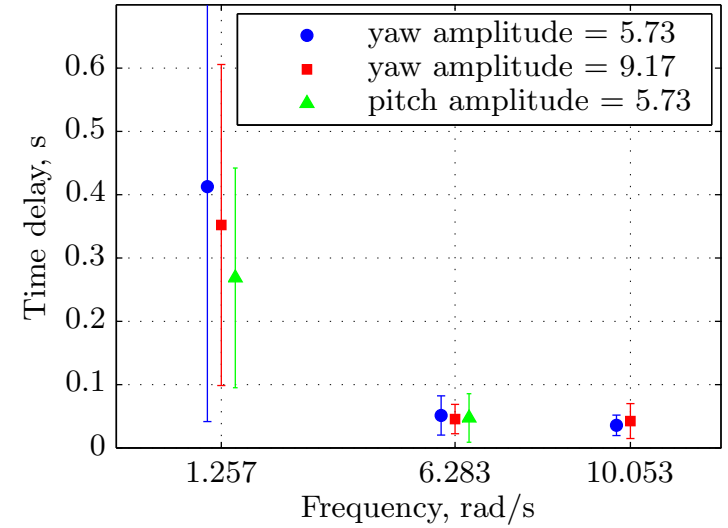

(a) Time-delay thresholds

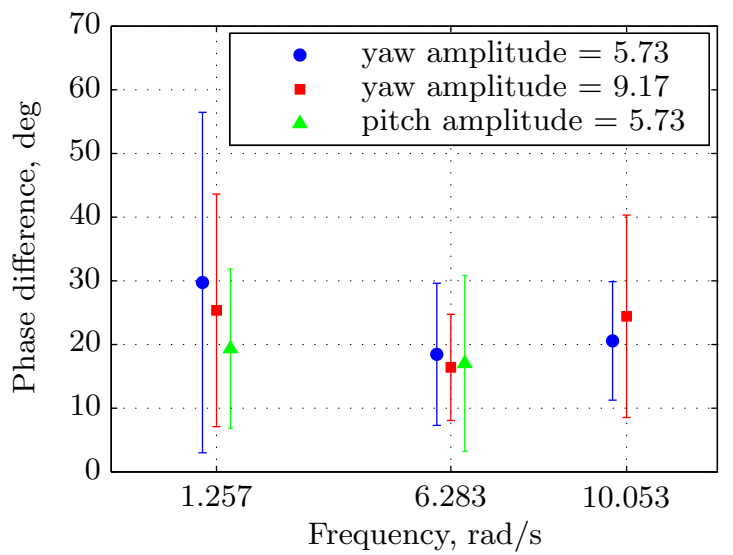

(b) Phase-error thresholds

Figure 14. Mean thresholds and standard deviations for all subjects and repetitions represented in terms of phase and time.

The time-delay thresholds (Figure 14(a)) show a clear decrease with frequency, as one might expect from a constant phase-error threshold that does not vary with frequency ( $c f$. Figure 7 ). In fact, observing the phase-error thresholds in Figure 14(b) no clear trend is visible. For equal amplitudes the mean phase-error threshold decreases slightly from the lowest to the mid frequency but rises again at the highest frequency. Whereas the lower amplitude also results in a lower threshold value for the lower frequencies, the opposite happens at the highest frequency. Pitch thresholds are lower than the corresponding yaw values for the lowest frequency. The lowest mean phase-error threshold measured was $17 \mathrm{deg}$ and the highest value was $30 \mathrm{deg}$. The grand mean of all conditions was 22 degrees.

The large standard deviation for the lower frequency conditions visible in Figure 14(b) was mainly caused by Subject 3, who had relatively high threshold values at these conditions. Figure 15 shows the results without Subject 3. Here it can be seen that the standard deviation is roughly the same for all frequencies. Since Subject 3 was very consistent throughout all repetitions, his or her data were not excluded and was used for all further analysis. 


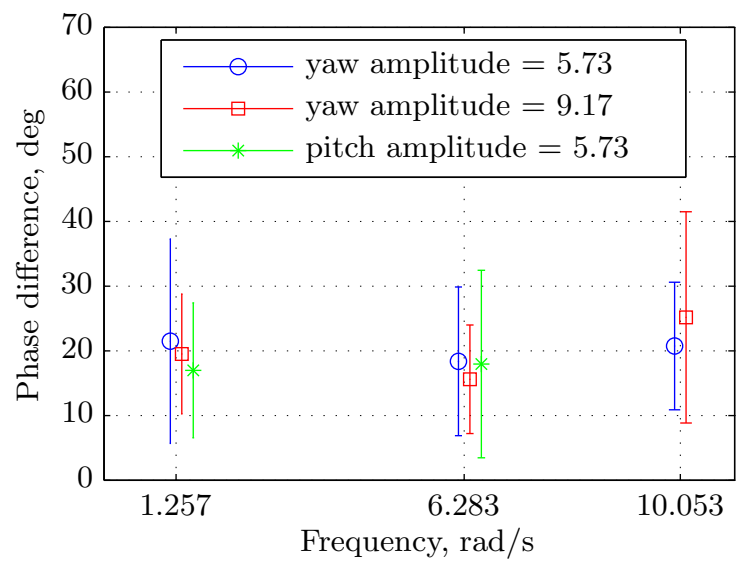

Figure 15. Means and standard deviations of all subjects and repetitions without Subject 3.

Figure 16 presents the mean of all conditions and repetitions separately for each subject. Here the relatively higher mean of Subject 3 is also noticeable. Moreover, there is a quite wide variation between subjects and the subjects with a higher mean threshold value also have a higher standard deviation.

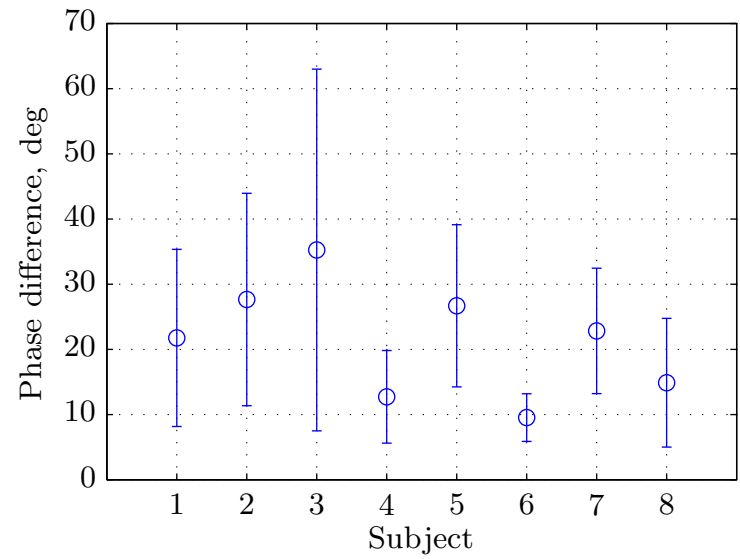

Figure 16. Means and standard deviations of all conditions per subject.

In Figure 17 both pitch conditions are plotted together with the corresponding values measured by Grant and Lee. ${ }^{4}$ As can be observed, the mean thresholds found in the present study were less than half of the ones found by Grant and Lee.

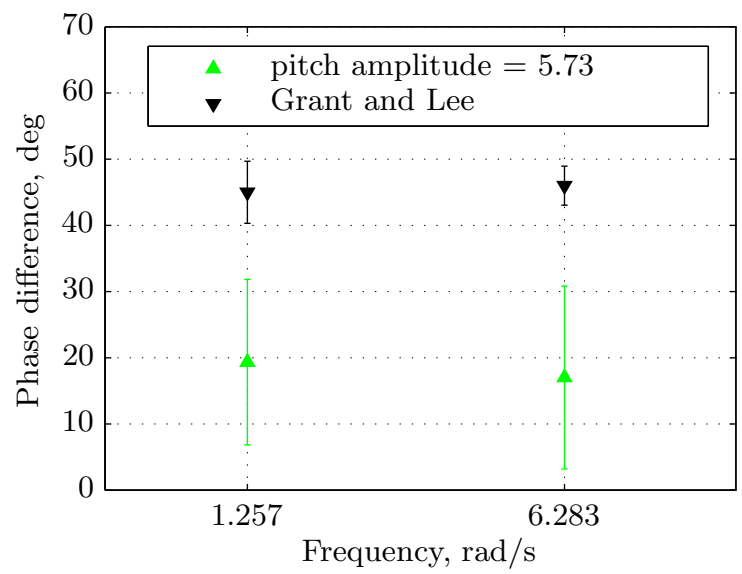

Figure 17. Means and standard deviations of all pitch conditions compared to data from Grant and Lee. ${ }^{4}$ 
Statictical analysis performed on the data showed no significant effect of amplitude or frequency in neither the yaw nor the pitch phase-error thresholds. Also, no significant differences were found between the pitch and the yaw thresholds.

An analysis of subjects comments shows that most subjects agreed that the high frequency conditions were the most difficult to judge. One of the reasons mentioned was the shorter time around the turnaround points ${ }^{a}$ which made it difficult to judge whether or not both stimuli were in phase. At the lowest frequency some subjects reported that they had difficulties to perceive any inertial motion. Especially Subject 3 reported this for many of the lower frequency conditions. Pitch conditions were, in general, considered to be slightly easier to judge than yaw conditions. However, subjects could not clearly describe why. Occasionally, some subjects would also mention that they thought that the amplitudes of the inertial and visual cues were not matching.

\section{Discussion}

An experiment was conducted to measure visual-vestibular phase-error detection thresholds. There were three independent variables: frequency, amplitude and axis of rotation. The results showed no significant effects of the main factors on the phase-error threshold.

The fact that the measured phase-error thresholds did not vary with frequency suggests that the mechanism for perception of visual-inertial cue synchronization relies on the detection of phase differences rather than on the detection of a pure time delay. Moreover, the hypothesis that the dynamics of the semicircular canals might influence the perceived phase difference between the two cues could not be confirmed. This result is in contrast to what has been found for the perception of amplitude mismatch, ${ }^{3}$ where the perceived coherence between inertial and visual cues was significantly affected by the stimulus frequency. However, it is in agreement with the data of Grant and Lee, ${ }^{4}$ which found a fairly constant phase-error threshold across frequencies for the condition with pitch motion amplitude of $5.73 \mathrm{deg} / \mathrm{s}$.

Comparing the actual threshold values found in this study with the ones from Grant and Lee ${ }^{4}$ it is clear that the pitch phase-error thresholds were much lower for the present work. The grand mean of the phase-error threshold in Grant and Lee's study was $57 \mathrm{deg}$ for all conditions and 41 deg for the conditions with an amplitude of $5.73 \mathrm{deg} / \mathrm{s}$ and it was as small as $22 \mathrm{deg}$ in the present investigation. Differences in the experimental methods used may be the cause of the discrepancy. In this study the threshold value was determined to be the phase-error at the $50 \%$ probability level in the psychometric curve. Grant and Lee used a $79 \%$ probability level. For a higher detection probability a higher phase-error vale may be expected.

Moreover, both studies were performed in different simulators and the visual systems used differed considerably. The influence of visual system characteristics, such as field-of-view, collimation, resolution and contrast, on the perception of cue synchronization should be further investigated to be able to interpret these results.

One other difference between the results of Grant and Lee and the ones presented here, was the absence of an influence of the stimulus amplitude on the phase-error thresholds. In their study they found that for the lowest amplitude the phase-error thresholds were relatively higher and were dependent on the stimulus frequency. At the lowest amplitude, the perception of inertial and visual motion may be more difficult, hindering the detection of phase errors. In the present experiment, since higher amplitudes were used, this effect might no longer be observable. Amplitudes higher than the lowest amplitude tested here $(5.73 \mathrm{deg} / \mathrm{s})$ may not significantly improve the detection of self-motion. This finding satisfies the assumption that the effect of amplitude would cease above a certain value.

The small differences found between the phase-error thresholds in yaw and pitch rejects the last hypothesis. Although the stimulation of the otolith organ was above the perception threshold, it did not seem to have a significant influence on the detection of visual-vestibular phase-errors at the amplitudes and frequencies tested. Perhaps at lower amplitudes, closer to the perception threshold, the extra cue due to otolith stimulation would play a bigger role.

The phase-error detection task was not an easy task, as also indicated by the large standard deviations registered for each subject. Visual-vestibular phase errors do not occur in everyday life and the concept of phase lead and lag is not as intuitive as amplitude mismatch, for example. There might have been a difference in strategy as well, with some subjects waiting until they clearly perceived the phase-error and others taking a general feeling that "something was wrong" as their threshold.

${ }^{a}$ the points of the motion profile where the velocity reaches zero. 
In further research, amplitude coherence zones results could be used to guarantee that subjects never have the feeling that there is an amplitude mismatch. The amplitude of the inertial signal could be adjusted depending on the signal frequency, in such a way that it would correspond to the point of mean coherence. Perhaps in this manner, the task would become easier and and the results more consistent across subjects.

\section{Conclusions}

An experiment was conducted to measure the influence of stimulus frequency and amplitude on the visual-vestibular phase-error detection thresholds. The measurements were made in yaw and pitch.

The measured thresholds suggest that humans act like phase-error detectors, rather than like time delay sensors and that for the range of amplitudes and frequencies tested, the mean phase-error thresholds is 22 degrees.

The amplitude and frequency of the stimulus did not affect phase error detection. These results are partially in agreement with the findings of Grant and Lee. ${ }^{4}$ The mean phase-threshold value, however, was much lower than the one found in that study. This difference might be attributed to the experimental methodologies use. The staircase method used here was designed to obtain a reliable threshold value at the $50 \%$ probability level of the psychometric curve, whereas in Grant and Lee the probability level was $79 \%$. Differences in the visual systems characteristics might have also played a role, but without further investigation no decisive conclusions can be drawn.

The measured thresholds for yaw were similar to the ones found in pitch. The stimulus amplitude was probably high enough above the angular motion sensory threshold to render the extra cue, the otolith stimulation, unnecessary.

If the results found here are to be directly applied to motion filter tuning in the SIMONA Research Simulator, then care should be taken that, for the range of amplitudes and frequencies tested, the angular inertial motion does not lead the visual cues by more than 22 degrees.

\section{Acknowledgments}

The second author is supported by a Toptalent grant from the Netherlands Organisation for Scientific Research (NWO).

\section{References}

\footnotetext{
${ }^{1}$ Gouverneur, B., Mulder, J. A., van Paassen, M. M., Stroosma, O., and Field, E. J., "Optimisation of the Simona Research Simulator's Motion Filter Settings for Handling Qualities Experiments," AIAA Modeling and Simulation Technologies Conference and Exhibit, No. AIAA 2003-5679, August 11-14 2003.

${ }^{2}$ van der Steen, F. A. M., Self-Motion Perception, Ph.D. thesis, Delft University of Technology, 1998.

${ }^{3}$ Valente Pais, A. R., van Paassen, M. M., Mulder, M., and Wentink, M., "Perception Coherence Zones in Flight Simulation," Journal of Aircraft, Vol. 47, No. 6, 2010, pp. 2039-2048.

${ }^{4}$ Grant, P. and Lee, P. T. S., "Motion-Visual Phase-Error Detection in a Flight Simulator," Journal of Aircraft, Vol. 44, No. 3, 2007, pp. 927-935.

${ }^{5}$ Chung, W. W. and Schroeder, J. A., "Visual and Roll-Lateral Motion Cueing Synchronization Requirements for MotionBased Flight Simulations," American Helicopter Society 53rd Annual Forum, Virginia Beach, VA, USA, April 29 - May 1, 1997, pp. 994-1006.

${ }^{6}$ Stahle, J., The Vestibular System, Almqvist \& Wiksell, 1984.

${ }^{7}$ Hosman, R. J. A. W. and van der Vaart, J. C., "Vestibular Models and Thresholds of Motion Perception. Results of Tests in a Flight Simulator," Internal Report LR-265, Delft University of Technology, Faculty of Aerospace Engineering, 1978.

${ }^{8}$ Fernandez, C. and Goldberg, J. M., "Physiology of peripheral neurons innervating semicircular canals of the squirrel monkey. II. Response to sinusoidal stimulation and dynamics of peripheral vestibular system," Journal of Neurophysiology, Vol. 34, No. 4, 1971.

${ }^{9}$ Fischer, M. and Kornmueller, A., "Optokinetisch ausgeloeste Bewegungswahrnehmung und optokinetischer Nystagmus," Journal fuer Psychologie und Neurologie, Vol. 41, 1930, pp. 273-308.

${ }^{10}$ Wertheim, A. H., Motion Perception During Self-Motion: The Direct Versus Inferential Controversy Revisited, Vol. 17, 1994, pp. 293-355.

${ }^{11}$ Howard, I. P., Human Visual Orientation, John Wiley and Sons Ltd, Chichester, United Kingdom, 1982.

${ }^{12}$ Levitt, H., "Transformed Up-Down Methods in Psychoacoustics," Journal of the Acoustical Society of America, Vol. 49, No. 2, 1971, pp. 467-477.

${ }^{13}$ Heerspink, H. M., Berkouwer, W. R., Stroosma, O., van Paassen, M. M., Mulder, M., and Mulder, J. A., "Evaluation of
} 
Vestibular Thresholds for Motion Detection in the SIMONA Research Simulator," AIAA Modeling and Simulation Technologies Conference and Exhibit, San Francisco, CA, USA, August 15-18, No. AIAA-05-6502, 2005.

${ }^{14}$ Berkouwer, W. R., Stroosma, O., van Paassen, M. M., Mulder, M., and Mulder, J. A., "Measuring the Performance of the SIMONA Research Simulator's Motion System," AIAA Modeling and Simulation Technologies Conference and Exhibit, San Francisco, CA, USA, August 15-18, No. AIAA-2005-6504, 2005.

${ }^{15}$ Stroosma, O., van Paassen, M. M., and Mulder, M., "Using the SIMONA Research Simulator for Human-machine Interaction Research," Proceedings of the AIAA Modeling and Simulation Technologies Conference and Exhibit, Austin, Texas, Aug. 11-14, 2003, No. AIAA-2003-5525, 2003, pp. 1-8.

${ }^{16}$ Stroosma, O., van Paassen, M. M., Mulder, M., and Postema, F. N., "Measuring Time Delays in Simulator Displays," Proceedings of the AIAA Modeling and Simulation Technologies Conference and Exhibit, Hilton Head, SC, USA, No. AIAA2007-6562, 2007 